\title{
Considering comorbidity in managing rheumatic diseases: going where trials cannot go
}

\author{
Daniel Aletaha* and Thomas Dörner
}

It remains a great challenge to treat the complexity of systemic rheumatic diseases in clinical practice. In contrast to what one may think, these challenges are not so much related to a lack of effective treatments, as many powerful drugs are available for treating rheumatoid arthritis, many of which became licensed over the course of the past decade [1]. The use of any of these new - as well as of the 'older' - drugs is scientifically based on results of randomized controlled trials. The classical design of these trials, however, is what makes the step into clinical practice so challenging. With the strict inclusion and exclusion criteria of these trials, the patient populations tested in those trials are in sharp contrast to patients requiring treatment in clinical practice. This is further supported by the fact that only a fraction of patients in a rheumatology practice would fulfill the criteria to enter phase II/III trials [2]. A central reason for this is that patients with potential risk factors for an adverse reaction to an investigative drug are excluded from the beginning to make the trial safer; but this group of patients constitutes a large portion of those who will ultimately require treatment in clinical practice. Until the recent calls for more pragmatic trials [3] that address patient populations with all the risk factors faced in daily practice have manifested in actual trial designs, physicians will often need to make their own decisions, weighing expected (but sometimes unknown) risks against the possible benefits.

Although a number of management guidelines for different rheumatic diseases, such as those by the European League Against Rheumatism (EULAR) or the American College of Rheumatology (ACR), are in the public domain, the guidance is inherently vague when it comes to the many different challenges of dealing with comorbid conditions in rheumatic diseases. The main concerns here are safety issues associated with treatments, such as in the case of nonsteroidal anti-inflammatory

*Correspondence: daniel.aletaha@meduniwien.ac.a

Division of Rheumatology, Division of Internal Medicine 3, Medical University Vienna, Waehringer Guertel 18-20, 1090 Vienna, Austria drug (NSAID) treatment in patients with liver or cardiovascular disease, but in theory one could also think about potential synergisms of treating the index disease and a comorbid condition at the same time - for example, when treating arthritis patients with concomitant inflammatory bowel disease.

The purpose of this new series of reviews in Arthritis Research and Therapy is to comprehensively provide an update on these issues, including new developments and current perspectives on rheumatic patients who present with a comorbid condition. Each review of this series therefore addresses a different organ or organ system. We are very grateful that experts specialized in rheumatology together with experts from other specialties have contributed to this series by reviewing the currently available literature combined with their expertise. Specifically, the series will provide a very broad perspective on distinct and frequent organ manifestations provided by experienced rheumatologists as well as pulmologists, nephrologists, oncologists, hematologists, infectious diseases specialists, and hepatologists, respectively, in each review.

The lack of clear recommendations on how to manage most of the comorbid situations, but also the difficulty of finding important data from cohort studies and registries, which are usually reported less prominently than clinical trials, are a major motivation for this series. The first of these reviews, which is published in the current issue of Arthritis Research and Therapy on 'Treating rheumatic patients with a malignancy' [4], is exemplary in supporting this motivation: malignancy, even 'just' by history, is a major challenge in real life, as many of the drugs used to treat rheumatic diseases are immunosuppressives, and as such are often claimed to increase the risk of malignancy, even in otherwise healthy individuals.

We hope that the reviews from the current comorbidity series will provide a comprehensive and up-to-date overview. Each review will allow the reader to gain a comprehensive look at the respective clinical question, and hopefully help to facilitate daily decisions in clinical practice. At the same time, academic centers are working on the research agenda to identify and fill gaps in the evidence on how to manage comorbidities in certain rheumatic diseases. Although these reviews do not 
constitute guidelines or recommendations, the current perspectives of specialists may promote what we are all striving for, optimal care in rheumatology.

This article is part of the series Comorbid conditions in subjects with rheumatic diseases, edited by Daniel Aletaha and Thomas Dörner.

Other articles in this series can be found at

http://arthritis-research.com/series/comorbid

\section{Competing interests}

The authors declare that they have no competing interests.

Published: 29 June 2011

\section{References}

1. Smolen JS, Aletaha D, Koeller M, Weisman MH, Emery P: New therapies for treatment of rheumatoid arthritis. Lancet 2007, 370:1861-1874.

2. Zink A, Askling J, Dixon WG, Klareskog L, Silman AJ, Symmons DP: European biologicals registers: methodology, selected results and perspectives. Ann Rheum Dis 2009, 68:1240-1246.

3. Boers M: A call for pragmatic treatment trials in rheumatoid arthritis. Nat Clin Pract Rheumatol 2008, 4:292-293.

4. Elandt K, Aletaha D: Treating rheumatic patients with a malignancy. Arthritis Res Ther 2011, 13:223.

doi:10.1186/ar3354

Cite this article as: Aletaha D, Dörner T: Considering comorbidity in

managing rheumatic diseases: going where trials cannot go. Arthritis Research \& Therapy 2011, 13:116. 